\title{
Intra and Interobserver Variability in the B-mode Transorbital Sonographic Measurement of the Optic Nerve Sheath Diameter in Healthy Nepalese Individuals
}

\author{
Ghimire $\mathbf{P}^{1}$, Chand BB ${ }^{2}$, Mahat YB ${ }^{1}$, Ojha $S^{1}$, Singh BP ${ }^{1}$ \\ ${ }^{1}$ Department of Radiology, Nepalgunj Medical College and Teaching Hospital, Kohalpur, Banke \\ ${ }^{2}$ Department of Radiology, Bheri Hospital, Banke, Nepal
}

Received: January 20, 2019

Accepted: April 30, 2019

Published: June 30, 2019

Cite this paper:

Ghimire P, Chand BB, Mahat YB, Ojha S, Singh BP. Intra and Interobserver Variability in the B-mode Transorbital Sonographic Measurement of the Optic Nerve Sheath Diameter in Healthy Nepalese Individuals. Nepalese Journal of Radiology 2019;9(13):18-23.https://doi.org/10.3126/njr.v9i1.24811

\begin{abstract}
Introduction: Ocular ultrasound has gained importance as a non-invasive imaging technique in the screening, diagnosis and treatment management of intracranial hypertension. The aim of our study was to estimate the intra and interobserver variability on B-mode ocular ultrasound measurement of optic nerve sheath diameter (ONSD).

Methods: ONSD of the right eye was measured by ultrasound examination among 120 healthy adult volunteers. The ONSD was assessed $3 \mathrm{~mm}$ behind the globe and two serial measurements were taken by two observers who were blinded to each other's findings.

Results: Age of the volunteers ranged from 18 to 27 years with a mean age of $23.07+/-$ SD of 2.486. The mean of ONSDs of two observers was $4.168+/-$ SD of $0.54 \mathrm{~mm}$. The intraclass correlation coefficient for first and second observers was 0.997 and 0.978 respectively at $95 \%$ confidence interval. The interobserver intraclass correlation coefficient was 0.977 at $95 \%$ confidence interval. No correlation of age and gender was noted with ONSD.
\end{abstract}

Conclusions: B-mode transorbital ultrasound measurement of ONSD is highly reproducible with significant intra-observer and inter-observer agreement. Ultrasound measurement of ONSD measurements can serve as indispensable tool in critical care.

Key words: Intracranial Hypertension; Interobserver; Intraclass

\section{INTRODUCTION}

Correspondence to: Dr. Prasanna Ghimire

Department of Radiology

Nepalgunj Medical College and Teaching Hospital

Kohalpur, Banke, Nepal

Email: drprasannaghimire@gmail.com
Point of care ultrasound has gained immense importance in emergency and critical care due to its wide availability, portability, non-invasive nature, low cost and due to possibility of performing bedside with real time assessment. ${ }^{1}$ There is a wide range of 
applications of ultrasound in critical care with significant positive decision making, overall management and prognostication. Ocular ultrasound has been integrated in the emergency and critical care for the diagnosis and assessment of intracranial hypertension. Optic nerve sheath diameter measurement has now been considered as surrogate marker for assessment of intracranial pressure in various studies. ${ }^{2}$ Unlike other cross-sectional imaging, ultrasound is however operator dependent which significantly impacts the sensitivity and specificity in diagnosis of various conditions. ${ }^{3}$ In this study, we aimed to study the intraobserver and interobserver variability in the ultrasonographic measurement of the optic nerve sheath diameter which has an impact on the overall management of patients with intracranial hypertension.

\section{METHODS}

This was a prospective observational study undertaken at Nepalgunj Medical College and Teaching Hospital, Banke, Nepal from June 2016 to May 2017 on healthy volunteers of greater than 18 years of age. The study was approved by the institutional ethics committee and verbal and written consent was obtained from all individuals prior to the enrollment to the study. Exclusion criteria were history of head injury, hypertension, endocrine abnormalities, ophthalmic and neurological problems.

Ultrasound was performed on Logiq P6 ultrasound machine (GE Healthcare, Waukesha, Washington) with a 7.5 MHz linear transducer for the assessment of the ONSD by two radiologists with greater than 5 years of experience. Volunteers were examined on supine position and were instructed to have a neutral gaze and the close the eyes. The transducer was then placed over the closed upper eye lid of the right eye after application of moderate amount of gel. The ONSDs were measured in the axial plane $3 \mathrm{~mm}$ behind the optic disc (Figure 1). The calipers were placed at the outer extent of the ONS perpendicular to its longitudinal axis. Two measurement were taken by each observer at 15 mins interval in two separate settings. The two observers were blinded to each other's findings.

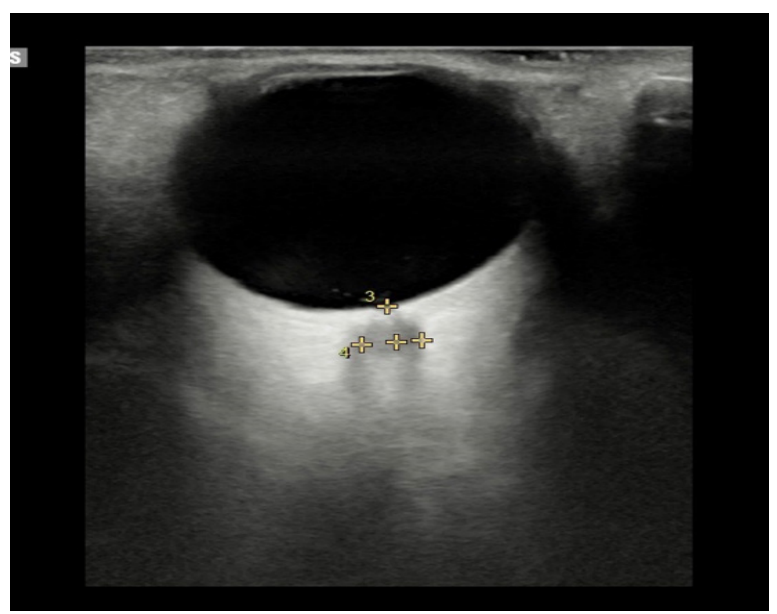

Figure 1: B-mode ultrasound measurement of Optic Nerve Sheath Diameter (ONSD), $3 \mathrm{~mm}$ behind the optic disc.

For all statistical analyses, SPSS Statistics Version 21.0 (IBM Corp., USA) was used. Intra-observer and interobserver reproducibility were calculated for each of the two observers on the basis of two serial measurements. Reproducibility was evaluated by means of the intraclass correlation coefficient (ICC).

A scatter plot was then constructed by plotting mean difference against mean of the two observers. The mean value and standard deviation (SD) of the mean differences were then calculated, and the SD used to describe the spread of measurement differences. The $95 \%$ confidence intervals of the mean difference constituted boundaries of the expected range of variability. Age and gender correlation were calculated by simple linear regression analysis and independent samples t-test.

\section{RESULTS}

A total of 120 healthy volunteers were included in the study (males 55\%, females $45 \%$ ). The age of the patients ranged from 18 to 27 years with a mean age of $23.07+/-\mathrm{SD}$ of 2.486 years. 
The minimum and maximum measured ONSDs was $2.50 \mathrm{~mm}$ and $4.90 \mathrm{~mm}$ with a mean of the ONSDs for the two measurements as $4.16+/-\mathrm{SD}$ of $0.55 \mathrm{~mm}$ for Observer 1 . For Observer 2, the minimum and maximum measured ONSDs was $2.50 \mathrm{~mm}$ and 4.90 $\mathrm{mm}$ with a mean of the ONSDs for two measurements as $4.17+/-\mathrm{SD}$ of $0.53 \mathrm{~mm}$. The mean of ONSDs of two observers was 4.168 $+/-\mathrm{SD}$ of $0.54 \mathrm{~mm}$ with the mean difference of two measurements being -0.0079 +/- SD of $0.0591 \mathrm{~mm}$. Scatter plot and agreement plot for ONSDs among the two observers is demonstrated in Figure 2 and Figure 3. The intraclass correlation coefficient for 1 st and 2nd observer was 0.997 and 0.978 respectively at $95 \%$ confidence interval (Table 1). The interobserver intraclass correlation coefficient was 0.977 at $95 \%$ confidence interval. For age, on simple linear regression analysis the $R$ value was $0.008, p=0.930$ and for gender $p=$ 0.532 at $95 \%$ CI.

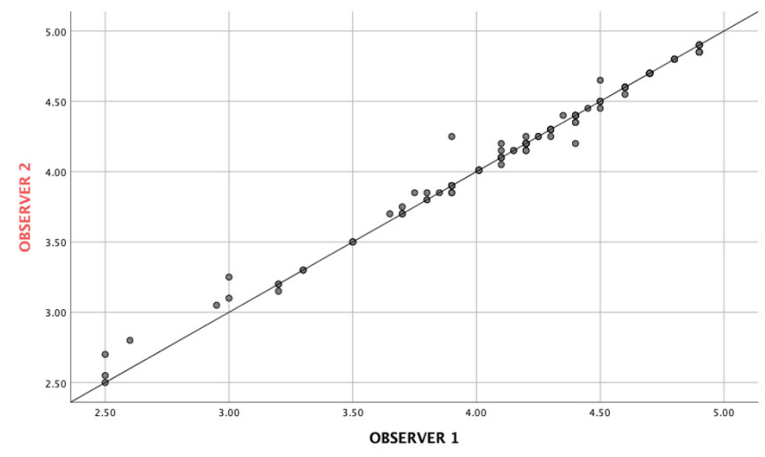

Figure 2: Scatter plot of Optic Nerve Sheath Diameter measurement shows observer 1 (x-axis) and observer 2 (y-axis), line of perfect agreement is shown.

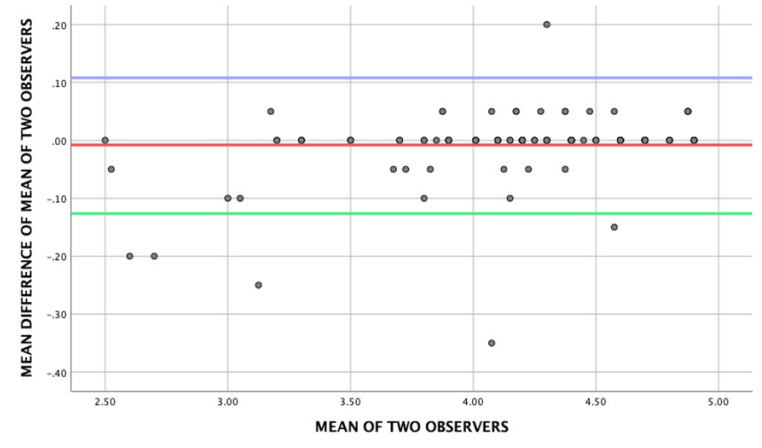

Figure 3: Agreement plot for ONSD made by two observers. Plots the mean difference of mean of two observers and mean of two observers.

\begin{tabular}{|c|c|c|}
\hline \multicolumn{3}{|c|}{$\begin{array}{l}\text { for intra-observer and inter-observer } \\
\text { measurement of optic nerve sheath } \\
\text { diameter of the right eve }\end{array}$} \\
\hline $\begin{array}{l}\text { OPTIC NERVE } \\
\text { SHEATH DIAM- } \\
\text { ETER OF THE } \\
\text { RIGHT EYE }\end{array}$ & $\begin{array}{l}\text { INTRA- } \\
\text { CLASS } \\
\text { CORRE- } \\
\text { LATION }\end{array}$ & $\begin{array}{l}\text { 95\% CON- } \\
\text { FIDENCE } \\
\text { INTERVAL }\end{array}$ \\
\hline \multicolumn{3}{|l|}{ INTRAOBSERVER } \\
\hline OBSERVER 1 & 0.997 & $0.995-0.998$ \\
\hline OBSERVER 2 & 0.978 & $0.968-0.984$ \\
\hline INTEROBSERVER & 0.977 & $0.967-0.984$ \\
\hline
\end{tabular}

\section{DISCUSSION}

Intracranial hypertension is a life-threatening condition with significant morbidity and mortality. ${ }^{4}$ A myriad of neurological as well as non-neurological conditions can lead to elevated intracranial pressure (ICP). An early recognition of the entity is warranted for management of such conditions. Various intracranial devices have been used for monitoring of intracranial pressure in emergency and intensive care units. ${ }^{3}$ Although these methods are accurate in measurement of ICP, they are however invasive in nature. Recognizable complications of these methods include procedure related as well as post procedural complications like bleeding, infection and even displacement of the various devices posing significant morbidity. ${ }^{5}$ Non-invasive surrogate techniques are thus instrumental in diagnosing as well as monitoring of increased ICP. $^{6}$ Various studies have demonstrated utility of imaging techniques in the overall management of these cases. Significant correlation has been noted with cross-sectional imaging such as Magnetic resonance imaging (MRI) and computed tomography findings of optic nerve sheath diameter (ONSD) with raised ICP. ${ }^{7}$ There are however various constraints and challenges with CT and MRI as most of these cases are critically ill patients with limited mobility and also many are on supportive devices that hinders assessment. Moreover, 
as individuals with raised ICP require regular monitoring, a potential hazard of radiation accounts for CT scan's decreased applicability. Ultrasound thus has gained immense utility in the emergency as well as intensive care units. Its undue advantage among the other imaging techniques is due to its easy and wide availability, real time assessment, portable and non-invasive nature. ${ }^{8}$ Ultrasound is often considered underutilized application with however a very wide potential in critical care including ocular pathology. ${ }^{3}$

Studies have demonstrated utility of ultrasound in patients with traumatic brain injury as well as in non- traumatic abnormalities. ${ }^{9}$ Ultrasound has been considered in the overall management of increased ICP from diagnosing, initiation and monitoring of treatment to identification of complications. ${ }^{10}$

Ultrasound, however has certain limitations in ocular ultrasound and measurement of ONSD. A multitude of factors influence the interpretation of ultrasonography. ${ }^{11}$ A number of neoplastic and non-neoplastic conditions affects the optic nerve. Recognition of these conditions prior to the ultrasound is warranted for proper assessment. It has been noted in various studies that intrinsic optic nerve pathology and any mass effect from extrinsic compression hinders and limits proper and accurate assessment of optic nerve.

Growing interest on the use of ultrasound as a proxy technique for measurement of ICP have been demonstrated by various studies. Many studies have shown correlation of ONSDs with ICP. ${ }^{12}$

As with other studies of observer agreement, in our study, we found intra-observer variability lesser than interobserver variability as well as excellent correlation for the measurements performed for intra-observer and interobserver agreement. ${ }^{13}$ High correlation values as in our study is to be expected. These results should however be interpreted cautiously as in our case only two observers were considered. Intraclass correlation coefficient must therefore be interpreted along with the $95 \%$ limit of agreement. This may differ when many individuals are involved in the sequential measurements when assessing response.

Since ultrasound is operator dependent, experience of the operator and proper technique are essential for assessment. The difference between raised ICP and normal ONSDs is minimal hence even a slight variation in the measurement leads to significant error in measurement. Ultrasound measurement of the ONSDs however have been shown to have acceptable intra-observer and interobserver variability as in our studies. Many studies have demonstrated a steep learning curve for measurement of ONSDs by novice operators trained with standardized protocols and often attain significant interobserver agreement with expert operators. ${ }^{14}$

There are various limitations to our study. Firstly, our study constitutes a relatively modest sample size which mandates study in a larger scale. Secondly, measurement was obtained by only two experienced observers. Besides, study was performed only in healthy volunteers and a reproducibility in patients with intracranial hypertension was not performed. However, it is promising that with standardized techniques, there is significant reliability and reproducibility in measurement of ONSD which has major implications in critical care management of raised intracranial pressure.

\section{CONCLUSION}

When ocular ultrasound is performed with standardized protocols for ONSD measurement, a significant intra-observer and interobserver agreement occurs thus demonstrating its reliability and 
reproducibility. Early recognition of increased intracranial pressure by ONSD measurement is pivotal in the overall management and monitoring of patients thereby reducing the overall morbidity and mortality.

\section{CONFLICT OF INTEREST}

None

\section{SOURCES OF FUNDING}

None

\section{REFERENCES}

1. Andruszkiewicz P, Sobczyk D. Ultrasound in critical care. Anaesthesiol Intensive Ther 2013;45(3):177-181. https://doi.org/10.5603/AIT.2013.0036

2. Toscano M, Spadetta G, Pulitano P et al. Optic Nerve Sheath Diameter Ultrasound Evaluation in Intensive Care Unit: Possible Role and Clinical Aspects in Neurological Critical Patients' Daily Monitoring. Biomed Res Int 2017;2017. https://doi.org/10.1155/2017/1621428

3. Hylkema C. Optic Nerve Sheath Diameter Ultrasound and the Diagnosis of Increased Intracranial Pressure. Crit Care Nurs Clin North Am 2016;28(1):95-99. https://doi.org/10.1016/j.cnc.2015.10.005

4. Munawar K, Khan MT, Hussain SW et al. Optic Nerve Sheath Diameter Correlation with Elevated Intracranial Pressure Determined via Ultrasound. Cureus 2019;11(2):11-12. https://doi.org/10.7759/cureus.4145

5. Jeon JP, Lee SU, Kim SE et al. Correlation of optic nerve sheath diameter with directly measured intracranial pressure in Korean adults using bedside ultrasonography. PloS One 2017;12(9):e0183170. https://doi.org/10.1371/journal. pone. 0183170
6. Roque PJ, Wu TS, Barth L et al. Optic nerve ultrasound for the detection of elevated intracranial pressure in the hypertensive patient. $A m \quad J$ Emerg Med 2012;30(8):1357-1363. https://doi.org/10.1016/j. ajem.2011.09.025

7. Shirodkar CG, Munta K, Rao SM, Mahesh MU. Correlation of measurement of optic nerve sheath diameter using ultrasound with magnetic resonance imaging. Indian $J$ Crit Care Med 2015;19(8):466-470. https://doi.org/10.4103/0972$\underline{5229.162465}$

8. Yuzbasioglu Y, Yuzbasioglu S, Coskun $S$ et al. Bedside measurement of the optic nerve sheath diameter with ultrasound in cerebrovascular disorders. Turk J Med Sci 2018;48(1):93-99. https://doi.org/10.3906/sag-1707-207

9. Pérez SS, Eichau SM, Rus MH et al. Usefulness of optic nerve ultrasound to predict clinical progression in multiple sclerosis. Neurologia (Barcelona, Spain) 2018. https://doi.org/10.1016/j.nrl.2017.12.009

10. Canakci Y, Koksal O, Durak VA. The value of bedside ocular ultrasound assessment of optic nerve sheath diameter in the detection of increased intracranial pressure in patients presenting to the emergency room with headache. Niger $J \quad$ Clin Pract 2018;21(6):778-782. https://doi.org/10.4103/njcp.njep 119 17

11. Zeiler FA, Ziesmann MT, Goeres $\mathrm{P}$ et al. A unique method for estimating the reliability learning curve of optic nerve sheath diameter ultrasound measurement. Crit Ultrasound $J$ 2016;8(1):9. https://doi.org/10.1186/s13089-016$\underline{0044-x}$ 
12. Rajajee V, Fletcher JJ, Rochlen LR, Jacobs TL. Comparison of accuracy of optic nerve ultrasound for the detection of intracranial hypertension in the setting of acutely fluctuating vs stable intracranial pressure: post-hoc analysis of data from a prospective, blinded single center study. Crit Care 2012;16(3):R79. https://doi.org/10.1186/CC11336

13. Popovic ZB, Thomas JD. Assessing observer variability: a user's guide. Cardiovasc Diagn Ther 2017;7(3):317-224. https://doi.org/10.21037/cdt.2017.03.12

14. Hassen GW, Bruck I, Donahue J et al. Accuracy of optic nerve sheath diameter measurement by emergency physicians using bedside ultrasound. $J$ Emerg Med 2015;48(4):450-457. https://doi.org/10.1016/j. jemermed.2014.09.060 